\title{
[gw22-e0145] OXIDISED LOW-DENSITY LIPOPROTEIN CHOLESTEROL AND THEIR RATIO IN THE DIAGNOSIS AND TREATMENT OF CORONARY HEART DISEASE
}

Huiling Huang, Weiyi Mai, Dan Liu, Yugang Dong The First Affiliated Hospital Of Sun Yat Sen University, Guangzhou, China

10.1136/heartjnl-2011-300867.394

Objective Oxidised LDL cholesterol (ox-LDL-C) is considered to be a key factor of initiating and accelerating atherosclerosis (AS). The purpose of this study is to observe the level in CAD (coronary artery disease) patients and Non-CAD patients. And we investigated the value of ox-LDL and oxidation ratio of LDL (ox-LDL/TC, ox-LDL/HDL-C and ox-LDL/LDL-C) in the diagnosis and prognosis of CAD patients. Futhermore, we aim to observe the effect of statins in the level of ox-LDL and oxidation ratio of LDL (ox-LDL/TC, ox-LDL/HDL-C and ox-LDL/ LDL-C), and discuss whether statins still have similar effect to ox-LDL in earlier period (within 2 weeks).

Methods and results Blood ox-LDL, total cholesterol (TC), high density lipoprotein cholesterol (HDL-C), low density lipoprotein cholesterol (LDL-C) and triglyceride (TG) were measured in patients with acute myocardial infarction (AMI, $\mathrm{n}=177$ ), unstable angina pectoris (UAP, $\mathrm{n}=195$ ), stable angina pectoris (SAP, $\mathrm{n}=228$ ), normal control $(n=120)$ and high risk control $(n=140)$. TC and TG were measured by enzymic method, HDL-C and LDL-C were measured by homogeneous method, and ox-LDL was measured by capture ELISA. Mean value of ox-LDL and oxidation ratio of $L D L$ is significantly higher in the CAD group than in the two control groups. The area under the curve (AUC) of receiver operating characteristic curve (ROC curve) is a criterium to evaluate the accuracy of diagnosing a disease. The AUC of ox-LDL, ox-LDL/TC, ox-LDL/HDL-C, ox-LDL/LDL-C and apoA1/apoB were more than $0.50(\mathrm{p}<0.001)$, while the AUC of TC, LDL-C, HDL-C were less than $0.50(p<0.001)$. Multivariate logistic regression analysis found that age and ox-LDL/LDL-C relate with short-term and long-term prognosis $(p<0.05)$. Furthermore, $584 \mathrm{CAD}$ patients received treatment of statins for 2 weeks. After treatment HDL-C and apoA1/ apoB were improved $10 \%$ and $14 \%$ respectively, TC, TG, LDL-C, ox-LDL, ox-LDL/TC, ox-LDL/HDL-C and ox-LDL/ LDL-C decreased $22 \%, 1.5 \%, 29 \%, 38 \%, 29 \%, 23 \%$ and $25 \%$ respectively. And the decrease of ox-LDL by statins is independent with LDL-C and TC.

Conclusions ox-LDL and oxidation ratio of LDL (ox-LDL/ TC, ox-LDL/HDL-C and ox-LDL/LDL-C) are closely related with AS, and they are better biomarkers for discriminating between patients with coronary artery disease and healthy 


\section{Abstracts}

subjects. Furthermore, they are valuable for prognosis in CAD patients. Statins can decrease the level of ox-LDL significantly, and the effect is independent with LDL-C and TC. 